\title{
Etalonnage de cristaux organiques : simulation par SHADOW
}

\author{
F. Legrand, G. Bridenne, G. Faussurier et M. Sanchez del Rio*
}

CEA, Service Conception Expérimentale de la Mesure, BP. 12, 91680 Bruyères-le-Châtel, France

* European Synchrotron Radiation Facility, BP. 220, 38043 Grenoble, France

\begin{abstract}
RESUME
Nous présentons ici les modifications apportées au programme BRAGG, utilitaire de SHADOW permettant le calcul de profils de diffraction produits par des cristaux organiques et plus généralement par n'importe quel cristal.

Nous avons vérifié la validité des modifications proposées en comparant les résultats obtenus avec la version antérieur du programme pour un cristal de silicium et avec des données tabulées pour un certain nombre de cristaux organiques (KAP, RbAP, TlAP, $\mathrm{PET}, .$. ).
\end{abstract}

\section{BUT}

Les spectrographes à haute résolution pour le rayonnement $X$ dans la gamme $0,5-5$ $\mathrm{keV}$ utilisent des cristaux organiques plans ou courbes tels que le béryl, les acides phtaliques (KAP, RbAP, TIAP, etc...) ou le PET. Le but de ce travail est de modéliser le comportement de tels cristaux mis en oeuvre dans des monochromateurs sur des lignes de lumière synchrotron à l'aide du programme SHADOW [1] et de ses utilitaires.

\section{LE PROGRAMME BRAGG2}

L'ensemble du groupe de programmes SHADOW a été développé pour l'analyse et le dessin de lignes de rayonnement synchrotron. Le programme utilise la technique du tracé de rayons. Ces lignes de lumière deviennent de plus en plus sophistiquées, ce qui oblige en permanence à développer et implanter dans le programme de nouveaux modèles en réponse à des problématiques successives.

Du point de vue de la modélisation de cristaux, les derniers développements de Bragg, pré-processeur de Shadow, permettent le calcul de courbes de diffraction de cristaux asymétriques et en transmission [2], mais une structure cristalline générale n'est pas encore prévue. C'est la raison pour laquelle nous proposons des modifications dans 'ce sens.

Bragg2 offre la possibilité de modéliser la réponse de cristaux comportant de 1 à 10 espèces atomiques arrangées en structures cubique, hexagonale, quadratique ou orthorhombique. Les positions des atomes sont introduites dans un simple fichier texte. Chaque utilisateur peut constituer sa propre bibliothèque de fichiers en fonction de ses besoins. 


\section{SIMULATIONS DE REPONSES DE CRISTAUX ORGANIQUES}

Nous avons effectué une série de validation en comparant les courbes de réflexion de cristaux organiques calculées par le programme avec celles des tables de Henke [3] et certains résultats expérimentaux enregistrés sur la ligne d'étalonnage de cristaux de l'anneau synchrotron Super-ACO (Orsay, France) [4]. Les figures 1 et 2 montrent la comparaison des résultats obtenus avec les données de Henke respectivement pour des cristaux de TIAP et RbAP.
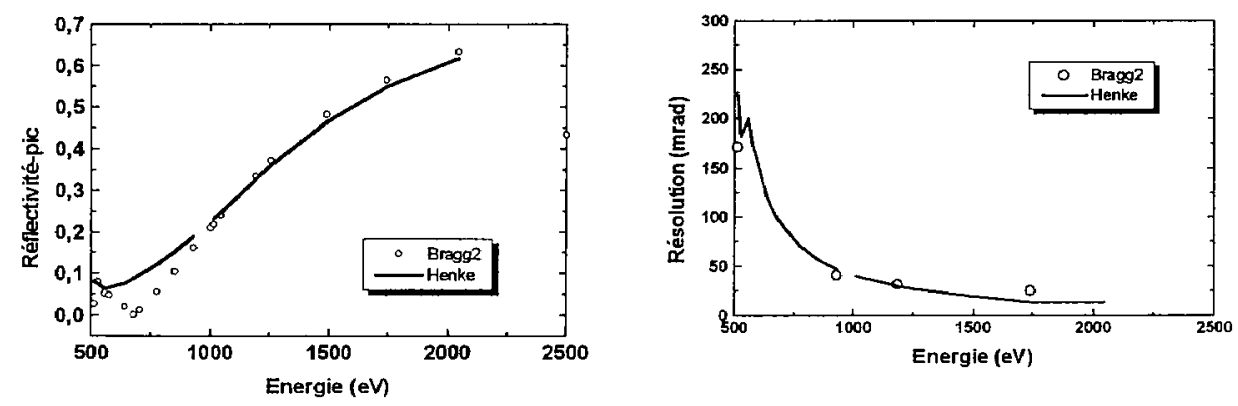

Fig. 1 : Comparaison des calculs de Bragg2 avec les valeurs tabulées par Henke pour le calcul de la réflectivité-pic et la résolution du TIAP [001]
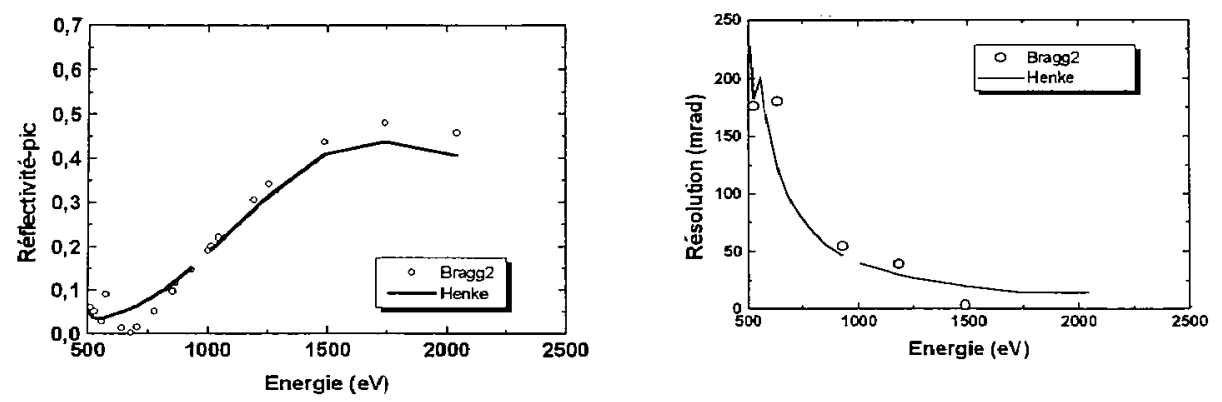

Fig. 2 : Comparaison des calculs de Bragg2 avec les valeurs tabulées par Henke pour le calcul de la réflectivité-pic et la résolution du RbAP [001]

\section{CONCLUSION}

Les résultats obtenus avec ce nouveau programme sont en bon accord avec les valeurs de référence.

Bragg2 permet donc de calculer la courbe de réflexion de nombreux cristaux. A cause des différences de formats dans les fichiers de résultats, il n'est pas encore possible d'utiliser ces pré-calculs dans les simulations par tracé de rayons de Shadow. Les modifications sont en cours.

[1] B. Lai \& F. Cerrina, NIM. A.246 (1986) 337

[2] M. Sanchez del Rio \& F. Cerrina, Rev. Sci. Instrum. 63 (1992) 936

[3] B.L. Henke, P. Lee, T.J. Tanaka, R. Shimabukuro \& B.K. Fujikawa. Atom. Data Nucl. Data Tables 27. Academic Press (1982)

[4] Beck \& al., " Etalonnage de cristaux organiques auprès du taisceau synchrotron de Super ACO", poster présenté à ce congrès 